\title{
Learning Dialog Policies from Weak Demonstrations
}

\author{
Gabriel Gordon-Hall* and Philip John Gorinski* \\ Huawei Noah's Ark Lab \\ London, UK \\ gabriel.gordon.hall@huawei.com \\ philip.john.gorinski@huawei.com
}

\author{
Shay B. Cohen \\ ILCC, School of Informatics \\ University of Edinburgh \\ scohendinf.ed.ac.uk
}

\begin{abstract}
Deep reinforcement learning is a promising approach to training a dialog manager, but current methods struggle with the large state and action spaces of multi-domain dialog systems. Building upon Deep Q-learning from Demonstrations (DQfD), an algorithm that scores highly in difficult Atari games, we leverage dialog data to guide the agent to successfully respond to a user's requests. We make progressively fewer assumptions about the data needed, using labeled, reduced-labeled, and even unlabeled data to train expert demonstrators. We introduce Reinforced Fine-tune Learning, an extension to DQfD, enabling us to overcome the domain gap between the datasets and the environment. Experiments in a challenging multi-domain dialog system framework validate our approaches, and get high success rates even when trained on outof-domain data.
\end{abstract}

\section{Introduction}

The dialog manager (DM) is the brain of a taskoriented dialog system. Given the information it has received or gleaned from a user, it decides how to respond. Typically, this module is composed of an extensive set of hand-crafted rules covering the decision tree of a dialog (Litman and Allen, 1987; Bos et al., 2003). To circumvent the high development cost of writing and maintaining these rules there have been efforts to automatically learn a dialog manager using reinforcement learning (RL; Walker 2000; Young et al. 2013). RL solves problems of optimal control - where past predictions affect future states - making it well-suited to dialog management, in which a misstep by the agent can throw the whole dialog off course. But using RL to train a dialog manager is not straightforward, and is often hindered by large dialog state spaces and sparse rewards (Gao et al., 2019).

\footnotetext{
*equal contribution
}

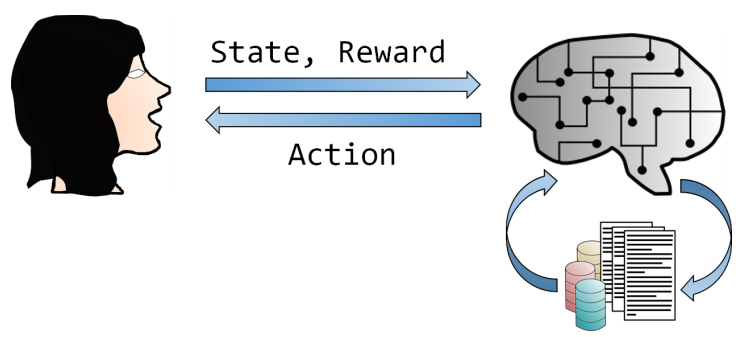

Figure 1: Illustration of reinforcement learning for dialog management. The agent (top right) interacts with the environment (left) by taking actions, and observing the resulting new state and reward. DQfD and RoFL RL agents are guided by an expert demonstrator (bottom right).

Neural network-based deep RL (Mnih et al., 2015) mitigates the problem of large state spaces (Fatemi et al., 2016; Li et al., 2017) but it still struggles when the DM has to choose a response or action - across multiple domains (e.g. hotel and flight booking). In addition, deep RL performs poorly without regular feedback - or reward - on the correctness of its decisions. In a dialog there is no obvious way to automatically quantify the appropriateness of each response, so RL training environments for dialog managers usually wait until conversation-end before assigning a reward based on whether the user's task, or goal, was completed.

An established way to deal with these difficulties is to guide the dialog manager with expert demonstrations during RL training (Lipton et al., 2018; Gordon-Hall et al., 2020), a high-level illustration of which is shown in Figure 1. This approach, however, requires a rule-based oracle to provide a suitable system response given a dialog state, and does not exploit the knowledge contained in the growing number of dialog datasets (Budzianowski et al., 2018; Rastogi et al., 2019).

In this paper, we address two key-questions that arise when training RL dialog agents with expert 
demonstrations: (i) Can we move away from rulebased experts and use weaker, cheaper demonstrations to guide the RL dialog manager? (ii) Can we exploit information gathered during RL training to improve the demonstrator and bridge the domain gap between dialog data and the RL environment?

To answer the first question, we explore three methods based on Deep Q-learning from Demonstrations (DQfD; Hester et al. 2017) that use trained experts derived from progressively weaker data. Our first and strongest expert is a Full Label Expert (FLE) trained on a labeled, in-domain dataset to predict the next system response. Second, we train a Reduced Label Expert (RLE) to predict the type of the next system response, but not its exact nature. Finally our third expert is a No Label Expert (NLE) that does not rely on any annotation at all, but is instead trained on unlabeled user utterance and agent response sentences. We show that all three experts can be used to successfully train RL agents, and two of them even allow us to train without expensive and often hard to come-by fully annotated in-domain dialog datasets.

We address our second key question - how to improve the experts during RL training - by presenting Reinforced Fine-tune Learning (RoFL), a fine-tuning algorithm inspired by Dataset Aggregation (DAgger; Ross et al. 2011). RoFL bridges the domain gap between dialog data and the RL environment by using the dialog transitions generated during training to update the expert's weights, adapting the previously learned knowledge to the learning environment. Our experiments show that RoFL training improves demonstrations gathered from the employed experts, giving a boost in RL performance and hastening convergence.

\section{Related Work}

Our work is closely related to research in using expert demonstrations to guide reinforcement learning dialog managers. Lipton et al. (2018) "spike" the deep Q-network (DQN; Mnih et al. 2015) replay buffer with a few successful demonstrations from a rule-based dialog manager. Gordon-Hall et al. (2020) extend this approach and apply Deep Q-learning from Demonstrations (DQfD) to dia$\log$, prefilling a portion of the buffer with expert transitions and encouraging the agent to imitate them by adding an auxiliary term to the DQN loss.

Demonstrations are not the only way to incor- porate external expertise into the dialog manager. One alternative is to use supervised learning to train a neural network policy on an in-domain dialog dataset, and then fine-tune it with policygradient RL on a user-simulator ( $\mathrm{Su}$ et al., 2016; Williams et al., 2017; Liu and Lane, 2017). Liu et al. (2018) fine-tune their RL policy on human rather than simulated users. Another, parallel, approach to RL-based DMs aims to increase the frequency of meaningful rewards. Takanobu et al. (2019) use inverse RL to learn a dense reward based on a dialog corpus, while Lu et al. (2019) decompose the task into subgoals that can be regularly assessed.

Weak demonstrations have been used outside of dialog system research to tackle RL environments with large state spaces and sparse rewards. Aytar et al. (2018) train an expert to imitate YouTube videos of people playing challenging Atari games and exceed human-level performance. Salimans and Chen (2018) beat their score on Montezuma's Revenge using only a single human demonstration, resetting the environment to different states from the expert trajectory. However we believe our work is the first to explore the use of weak demonstrations for DQfD in a dialog environment.

RoFL, our proposed fine-tuning method, is inspired by DAgger (Ross et al., 2011), an iterative imitation learning algorithm that incorporates feedback from an expert to improve the performance of a policy. DAgger requires an on-line expert that can be queried at any time, and which bounds the policy's performance. If the expert is suboptimal the policy will be too. Chang et al. (2015) lift this restriction, allowing the policy to explore the search space around expert trajectories, but their method (LOLS) does not incorporate RL policy updates as we do.

\section{Background}

Training a dialog manager - or agent - with reinforcement learning involves exposing it to an environment that assigns a reward to each of its actions. This environment consists of a database that the DM can query, and a user-simulator that mimics a human user trying to achieve a set of goals by talking to the agent. The more user goals the agent satisfies, the higher its reward. Given the current state $s_{t}$ of the dialog, the agent chooses the next system action $a_{t}$ according to a policy $\pi$, $a_{t}=\pi\left(s_{t}\right)$, and receives a reward $r_{t}$. The ex- 
pected total reward of taking an action $a$ in state $s$ with respect to $\pi$ is estimated by the Q-function:

$$
\begin{gathered}
Q(s, a)=\mathbb{E}_{\pi}\left[\sum_{k=0}^{T-t} \gamma^{k} r_{t+k} \mid s_{t}=s, a_{t}=a\right] \\
\pi^{*}(s)=\underset{a \in A}{\arg \max } Q^{*}(s, a)
\end{gathered}
$$

where $T$ is the maximum number of turns in the dialog, $t$ is the current turn, and $\gamma$ is a discount factor. The policy is trained to find the optimal Q-function $Q^{*}(s, a)$ with which the expected total reward at each state is maximized. $\pi^{*}(s)$ is the optimal policy obtained by acting greedily in each state according to $Q^{*}$ (Sutton and Barto, 2018).

Deep Q-network (DQN; Mnih et al. 2015) approximates $Q(s, a)$ with a neural network. The agent generates dialogs by interacting with the environment, and stores state-action transitions in a replay buffer in the form $\left(s_{t}, a_{t}, r_{t}, s_{t+1}\right)$. Rather than always acting according to its policy $\pi$, an $\epsilon$-greedy strategy is employed in which the agent sometimes takes a random action according to an "exploration" parameter $\epsilon$. Transitions aggregated in the replay buffer are sampled at regular intervals and used as training examples to update the current estimate of $Q(s, a)$ via the loss:

$$
\begin{gathered}
y_{t}=r_{t}+\gamma \max _{a^{\prime}} Q\left(s_{t+1}, a^{\prime} ; \theta^{\prime}\right) \\
\mathcal{L}(Q)=\left(y_{t}-Q\left(s_{t}, a_{t} ; \theta\right)\right)^{2}
\end{gathered}
$$

where $\theta^{\prime}$ are the fixed parameters of a target network which are updated with the current network parameters $\theta$ every $\tau$ steps, a technique which improves the stability of DQN learning.

Deep Q-learning from Demonstrations (DQfD; Hester et al. 2017), an extension to DQN, uses expert demonstrations to guide the agent. DQfD, prefills a portion of the replay buffer with transitions generated by the expert. The agent learns to imitate these demonstrations by augmenting $\mathcal{L}(Q)$ with an auxiliary loss term $\mathcal{L}_{\text {aux }}(Q)$ :

$$
\mathcal{L}_{\mathrm{DQfD}}(Q)=\mathcal{L}(Q)+\mathcal{L}_{\text {aux }}(Q)
$$

The term $\mathcal{L}_{\text {aux }}$ depends on the expert used to provide demonstrations. For each of our three experts we will define a different auxiliary loss.

\section{Method}

It has been shown that DQfD successfully trains a dialog manager when its demonstrations come from either a rule-based, or strong pre-trained expert (Gordon-Hall et al., 2020). To avoid writing rules, and to exploit the knowledge contained in external datasets, we expand on previous work and adapt DQfD for use with three progressively weaker and cheaper experts. Furthermore, we introduce our RoFL algorithm, describing how we fine-tune the expert during RL training.

Full Label Expert We define a Full Label Expert (FLE) as a classifier trained on a human-tohuman in-domain dialog dataset to predict, given the conversation state, the next action. For such an expert, the action space of the dataset corresponds to the actions in the RL environment and, as a result, we can use the original DQfD large margin classification term as an auxiliary loss:

$$
\begin{aligned}
\mathcal{L}_{\text {aux }}(Q)= & \max _{a \in A}\left[Q(s, a)+\ell\left(a_{E}, a\right)\right] \\
& -Q\left(s, a_{E}\right)
\end{aligned}
$$

where $a_{E}$ is the action the expert took in $s$, and $\ell\left(a_{E}, a\right)$ is 0 when the agent's chosen action is the same as the action taken by the expert demonstrator, and a positive constant $c$ otherwise:

$$
\ell\left(a_{E}, a\right)= \begin{cases}0, & \text { if } a=a_{E} \\ c, & \text { otherwise }\end{cases}
$$

This FLE approach is similar to the data-driven expert introduced by Gordon-Hall et al. (2020).

Reduced Label Expert A Full Label Expert is trained on fully-annotated in-domain data, but this is lacking for many domains, and is expensive to collect and label from scratch (Shah et al., 2018). However, although existing dialog datasets often differ in annotation, many share high-level system labels: inform and request. inform actions denote that the system provides information; request actions that the system asks for it. A system utterance from a hotel-booking dataset, e.g. "The Le Grand Hotel costs $\$ 48$ per night, how many nights do you want to stay?", could be labelled: [hotel-inform-price, hotel-request-duration], while a sentence from a taxi-booking dataset, e.g. "Please let me know the dropoff location.", could be annotated: taxi-request-dropoff. Although 


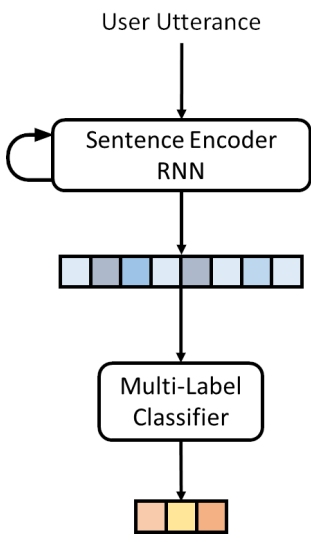

Figure 2: Reduced Label Expert (RLE) architecture.

the domain and type of information are different, all actions $\mathcal{A}$ in either dataset can be broadly partitioned into sets $A_{\text {reduced }} \subset \mathcal{A}$ according to whether they inform, request, or do both.

We introduce a Reduced Label Expert (RLE) to take advantage of this common annotation format across diverse datasets. The RLE is a multilabel classifier that predicts the high-level annotation set $A_{\text {reduced }}$ - or reduced label - of the next system action given the list $s_{N L}$ of the last few utterances in the dialog. The RLE is trained on a dialog dataset stripped down to inform, request, and other (for all other actions) annotations. Its architecture is outlined in Figure 2. The previous user utterances are passed through a recurrent encoder, for example an RNN. The final hidden state of the encoder is then passed through a multi-label classifier which uses the sigmoid function to score each reduced label.

Once trained, we use the RLE to guide the dialog manager during DQfD training. First we divide all environment actions into reduced label sets. For example, the inform set would consist of the environment actions that pertain to providing information to the user. Unlike the FLE, the RLE does not predict exact actions, so we uniformly sample an environment action from the predicted reduced label set $a_{E} \sim A_{\text {reduced }}$ to use as an expert demonstration when prefilling the replay buffer. For example, if the RLE predicts request the expert might take the action request-hotel-price. In order to use the expert in network updates, we reformulate the $\ell$ term in the DQfD's auxiliary loss to account for the expert's reduced label prediction:

$$
\ell\left(A_{r d c d}, s_{t}\right)= \begin{cases}0, & \text { if } \pi_{\theta}\left(s_{t}\right) \in A_{r d c d} \\ c, & \text { otherwise }\end{cases}
$$

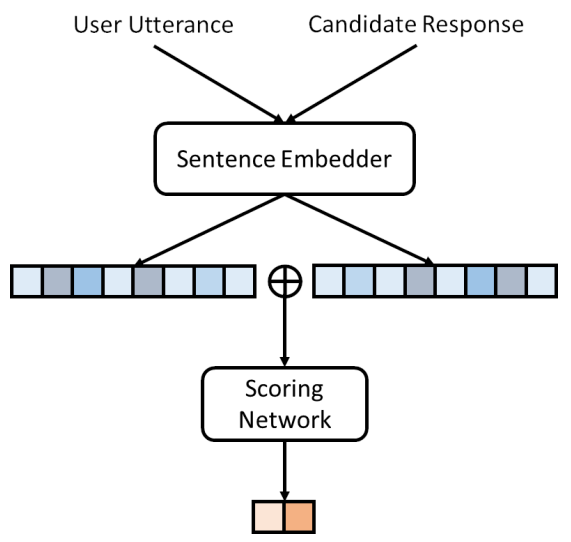

Figure 3: No Label Expert (NLE) architecture.

$$
A_{r d c d}=R L E\left(s_{N L}\right)
$$

The agent is penalized by a positive constant term $c$ if the action predicted by its current policy $\pi_{\theta}$ is not in the set of actions licensed by the RLE.

No Label Expert While the RLE enables the use of data not annotated for the target dialog environment, it still requires labeled dialog data. This raises the question: can we employ an expert that does not rely on annotations at all?

To address this challenge, we propose a $\mathrm{No} \mathrm{La}$ bel Expert (NLE) that uses an unannotated dialog dataset consisting of pairs of sentences $\left(s_{u}, s_{a}\right)$, representing user utterances and the corresponding agent responses. The goal of the NLE is to predict whether, for a given pair of sentences, $s_{a}$ is an appropriate response to $s_{u}$. In this regard, it resembles models used to predict textual inference (Bowman et al., 2015). The NLE architecture is outlined in Figure 3. The previous user utterance and a verbalized system response - generated by an NLG component - are consecutively passed through a sentence embedder. Their encodings are then concatenated and passed through a network which scores how appropriate the response is given the utterance.

The NLE is trained on unannotated human-tohuman dialog datasets which are formatted into pairs of user utterances and agent responses. We treat these as positive instances, making the tacit assumption that in the data the agent's reply is always relevant given a user utterance. As a result, the data lacks negative examples of irrelevant agent responses. This can be mitigated by artificially creating negative pairs $\left(s_{u}, s_{a}^{\prime}\right)$ from the original data by pairing each user utterance $s_{u}$ 
with random agent sentences $s_{a}^{\prime}$, drawn uniformly from all agent responses that were not observed for the original $s_{u}$. Given such a dataset of positive and negative user-agent interactions, we train an NLE that learns to output 1 if a system response corresponds to the last user utterance, and 0 if it does not. Once trained, we use this NLE to guide the DQfD dialog manager.

When prefilling the replay buffer with expert demonstrations, we calculate the set $A_{\text {no label }}$ of all actions $a$ whose verbalization $s_{a}$ leads to an NLE output that exceeds a threshold $\rho$ when taken as a response to the last user utterance $s_{u}$. We then use a random action from this set $a_{E} \sim A_{\text {nolabel }}$ as the expert demonstration and place it in the replay buffer. We use a similar $\ell$ term in the auxiliary loss to the Reduced Label Expert, which penalizes the agent if the action $a$ predicted by its current policy is not in the set of actions licensed by the expert, i.e., if $a \notin A_{\text {nolabel }}$ :

$$
\begin{gathered}
\ell\left(A_{\text {nolbl }}, s_{t}\right)= \begin{cases}0, & \text { if } \pi_{\theta}\left(s_{t}\right) \in A_{\text {nolbl }} \\
c, & \text { otherwise }\end{cases} \\
A_{\text {nolbl }}=\left\{a \mid N L E\left(\left[s_{u} ; s_{a}\right]\right)>\rho\right\}
\end{gathered}
$$

where $\rho$ is between 0 and 1 and $c$ is a positive constant penalty factor.

Domain Adaptation through Fine-tuning We train our experts on dialog datasets created by humans talking to humans. This data is necessarily drawn from a different distribution to the transition dynamics of an RL environment. In other words, there is a domain gap between the two.

We seek to narrow this gap by introducing Reinforced Fine-tune Learning (RoFL): For $d$ pretraining steps, transitions are generated according to a weak expert policy $\pi^{\xi_{\phi}}$, where the weak expert $\xi$ has parameters $\phi$. If a transition's reward exceeds a threshold $t h$, we treat it as in-domain data and add it to a buffer $\mathcal{D}$. Every $\eta$ steps the expert is fine-tuned on the in-domain data gathered so far and its parameters are updated. At the end of pretraining the final fine-tuned expert's weights are frozen and its policy is used to generate demonstration transitions for another $d$ steps. This ensures that the permanent, demonstration portion of the replay buffer is filled with transitions from the fine-tuned expert. RoFL is agnostic to the expert in question and we apply it to each of our methods described above.

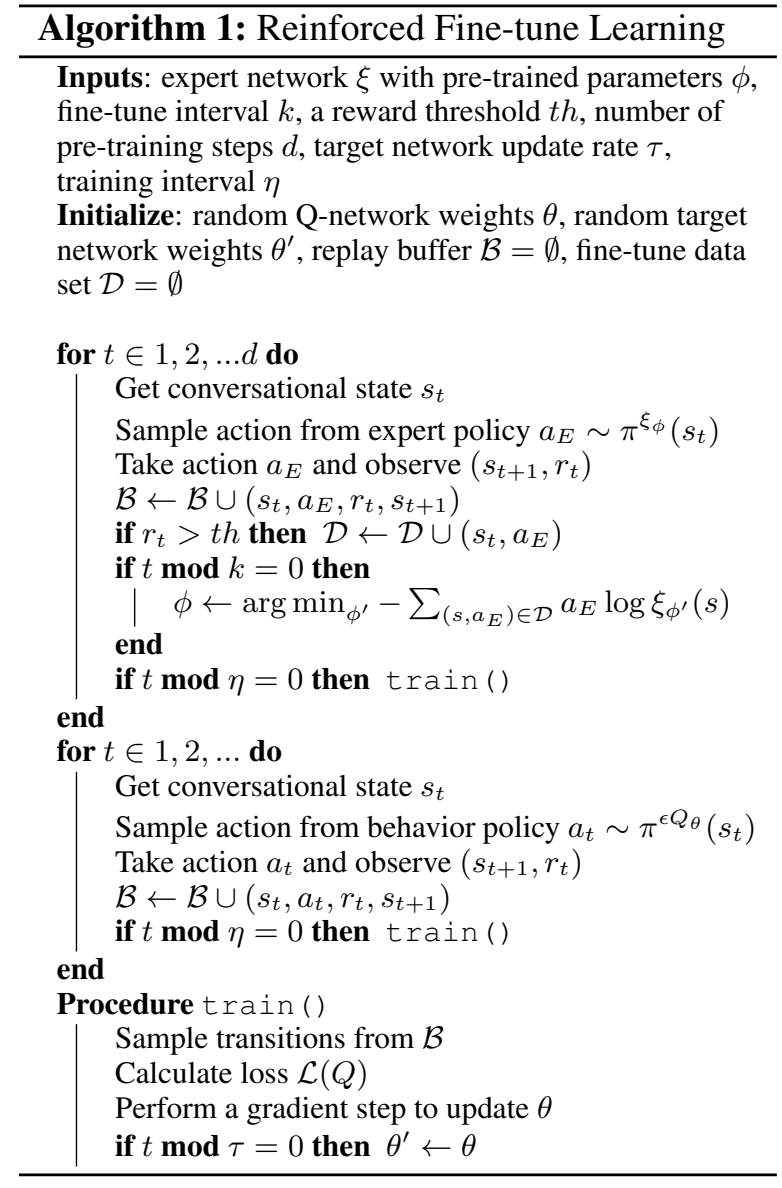

\section{Experimental Setup}

We evaluate our weak experts in ConvLab (Lee et al., 2019), a multi-domain dialog framework based on the MultiWOZ dataset (Budzianowski et al., 2018). In ConvLab, the dialog manager's task is to help a user plan and book a trip around a city, a problem that spans multiple domains ranging from recommending attractions for sightseeing, to booking transportation (taxi and train) and hotel accommodation.

ConvLab supports RL training with an environment that includes an agenda-based user-simulator (Schatzmann et al., 2007) and a database. The agent has a binary dialog state that encodes the task-relevant information that the environment has provided so far. This state has 392 elements yielding a state space of size $2^{392}$. In each state there are 300 actions that the DM can choose between, corresponding to different system responses when verbalized by the Natural Language Generation (NLG) module. These actions are composite and can consist of several individual informs and requests. For example, [attraction-inform-name, 
attraction-request-area] is one action.

We train our DMs on the exact dialog-acts produced by the user-simulator, avoiding error propagation from a Natural Language Understanding (NLU) module. We use ConvLab's default template-based NLG module to verbalize system actions when using the RLE and NLE.

First, we experiment with experts trained on the in-domain MultiWOZ dataset ${ }^{1}$. For the FLE we train on the full annotations; for the RLE we reduce the annotations to minimal inform, request, other labels; and for the NLE we only use the unannotated text. We also experiment with experts trained on out-of-domain (OOD) data. To this end, we combine two datasets: Microsoft E2E (Li et al., 2018) - 10,087 dialogs composed of movie, restaurant and taxi booking domains - and Maluuba Frames (El Asri et al., 2017) which is made up of 1,369 dialogs from the flight and hotel booking domains. While three of these domains are also in MultiWOZ, the specifics of the conversations are different.

Our Full Label Expert is a feedforward neural network (FFN) with one 150 dimensional hidden layer, ReLU activation function and 0.1 dropout which takes the current dialog state as input. The Reduced Label Expert uses the last utterance in the conversation as context, which is embedded with 300 dimensional pre-trained GloVe embeddings (Pennington et al., 2014), then passed through a uni-directional 128 dimensional hidden layer GRU (Cho et al., 2014) from which the last hidden state is used to make a multi-label prediction. Finally, our No Label Expert uses pre-trained BERT $_{\text {base-uncased }}$ (Devlin et al., 2018) to embed and concatenate user and agent utterances into 1536dimensional input vectors, and employs a feedforward neural network with SELU activations (Klambauer et al., 2017) to predict whether the agent's response is an appropriate answer to the last user utterance. Note that the RLE and NLE both take natural language as input yet use different word embeddings. We conducted preliminary experiments to evaluate the efficacy of BERT and GloVe embeddings for the respective expert training tasks. While we found that the NLE greatly benefited from BERT over GloVe, the RLE performance did not differ between embeddings. Since GloVe vectors yield a significant runtime advantage over the course of RL training, we used GloVe

\footnotetext{
${ }^{1}$ We use MultiWOZ2.0 with ConvLab user annotations
}

for the RLE, while employing slower BERT embeddings for the NLE due to the significantly better performance.

For RL training of our DQfD agents, we use a prioritized replay buffer (Schaul et al., 2015) with a maximum buffer size of 100,000 transitions. We follow the DQfD setup of (Gordon-Hall et al., 2020) and apply L2 regularization with a weight of $10^{-5}$ and drop the $n$-step term from the original DQfD loss. All RL networks have a 100 dimensional hidden layer, a dueling network structure, and use the double DQN loss (Wang et al., 2015; Van Hasselt et al., 2016). All our networks are trained with the RAdam optimizer (Liu et al., 2019) with a learning rate of 0.01 . For a complete list of hyperparameters used for our experiments refer to the attached Supplemental Material.

We slightly alter the RoFL algorithm presented in 4 to account for the fact that ConvLab only rewards the agent based on whether it successfully completed the task at the end of a dialog (intermediate steps are uniformly assigned a -1 step penalty). Rather than immediately adding transitions to the fine-tune dataset $\mathcal{D}$, we wait until the end of a conversation and check if its total reward exceeds a threshold $t h$. If it does, we assume that all transitions in that conversation are perfect, and add them to $\mathcal{D}$. For our experiments we empirically determine $t h$, and set it to 70 .

We train all our RL-based dialog managers for 3 sessions of 2,500,000 steps, and anneal the exploration parameter $\epsilon$ over the first 500,000 to a final value of 0.01. Results and training graphs in the following section are the average of these 3 sessions. Each session takes under 10 hours on one NVIDIA GeForce RTX 2080 GPU. We compare our approach to supervised and reinforcement learning baselines.

\section{Results}

Table 1 shows evaluation results over 1,000 dialogs for baseline and DQfD dialog managers using our three proposed experts inside ConvLab's evaluation environment. The Rule baseline is a rule-based DM included in ConvLab. FFN is a supervised learning baseline DM that directly uses the same in-domain classifier introduced in Section 4 to predict the next action. It is trained on MultiWOZ, and achieves $21.53 \%$ accuracy on the test set. Deep Q-network (DQN) is an RL agent which uses the hyperparameters described 


\begin{tabular}{l|c|c|c|c}
\hline & Turns & Inform & Match & Success \\
\hline Rule & 5.25 & 94.00 & 100 & 100 \\
FFN & 11.67 & 81.00 & 52.63 & 61.00 \\
DQN & 18.79 & 28.50 & 11.07 & 11.85 \\
PPO & 5.79 & 65.67 & 72.51 & 63.27 \\
\hline RE & 5.33 & 92.33 & 97.07 & 98.33 \\
FLE & 6.81 & 89.67 & 94.12 & 91.67 \\
RLE & 7.64 & 81.33 & 89.34 & 85.03 \\
NLE & 7.20 & 84.67 & 85.31 & 86.83 \\
\hline FFN-ft & 9.62 & 83.00 & 90.79 & 76.00 \\
FLE+R & 6.75 & $\mathbf{9 0 . 0 0}$ & $\mathbf{9 4 . 5 7}$ & 92.47 \\
RLE+R & $\mathbf{6 . 3 8}$ & 88.67 & 90.62 & $\mathbf{9 2 . 9 3}$ \\
NLE+R & 6.89 & 89.00 & 92.68 & 91.00 \\
\hline
\end{tabular}

Table 1: Evaluation results of baseline systems (top) as well as DQfD with rule-based and our weak expert approaches trained in-domain. The middle section denotes DQfD agents trained without RoFL; the bottom section shows results for agents trained with RoFL. Evaluation is conducted using an agenda-based user-simulator for 1000 dialogs. Reported scores are average number of Turns, Inform F1, Match Rate, and Success Rate. Best performing weak expert agents are in bold.

in Section 5 except that it does not use demonstrations. We also compare against an agent trained with Proximal Policy Optimization (PPO; Schulman et al. 2017), an actor-critic based RL algorithm widely used across domains. We use the PPO hyperparameters laid out in Takanobu et al. (2019). The middle third of Table 1 summarizes results for DQfD agents trained with rule-based (RE), Full Label (FLE), Reduced Label (RLE), and No Label (NLE) experts. The bottom third shows results for our weak expert methods trained with RoFL (+R). We follow Takanobu et al. (2019) and report evaluation results in terms of average dialog length (Turns), F1-Score of the information provided that was requested by the user, Match Rate of user-goals, and Success Rate - the percentage of dialogs in which all information has been provided and all booking information is correct.

As expected, the Rule agent - written specifically for ConvLab - almost perfectly satisfies user goals. FFN is considerably worse, with a $40 \%$ lower Success Rate, and half the Match Rate of the rule-based agent. For standard DQN, the environment's large state and action spaces pose a serious challenge, and it barely exceeds $11 \%$ Success and Match Rates. PPO achieves a respectable
$63 \%$ success rate, outperforming the FFN baseline. Crucially, all DQfD agents significantly outperform the FFN, DQN, and PPO baselines, with the RE and FLE approaches coming within 3\% and $6 \%$ respectively of the Rule agent's performance.

In the remainder of this section we will further analyze and compare the performances of DQfD agents with progressively weak demonstrations using in-domain and out-of-domain experts, as well as those trained with and without RoFL.

In-Domain Weak Expert DQfD We train indomain reduced and no label experts on the MultiWOZ dataset. The RLE scores $77 \mathrm{~F} 1$ on the reduced label test set, while the NLE manages 71 F1 of predicting whether an agent response belongs to a user utterance on the unannotated test set. As shown in Table 1 (middle), the scores of DQfD agents with in-domain experts follow a clear trend corresponding to the type of demonstration data. After 2.5 million training steps, the FLE - with the most informative demonstrations - clearly outperforms both RLE and NLE methods, while the latter two perform similarly.

Figure 4 shows graphs of the average Success Rates of DQN, PPO, and our proposed DQfD agents over the course of training. DQN struggles to find successful dialog strategies, although its Success Rate slowly inclines and seems to gain some traction towards the end of the maximum training steps. To begin with PPO learns rapidly, faster than RLE and NLE, but its Success Rate plateaus in the $60 \%$ range; it seems to learn to end dialogues too early. Both RE and FLE start with performance advantages, due to their high quality expert demonstrations. Over time, RE even approaches the Success Rate of its rule-based expert demonstrator. The FLE consistently outperforms approaches with weaker demonstrations, quickly exceeding the Success Rate of the underlying FFN after an early dip when the agent's exploration parameter $\epsilon$ is relatively high.

The NLE comfortably outperforms the Reduced Label Expert throughout training, with the RLE only overtaking it at the end. We believe that this strong relative performance makes sense if we consider that, during pre-training, the NLE acts according to a more fine-grained action set than the RLE. While the RLE partitions the actions according to their reduced label, these sets are broad and contain many irrelevant responses, whereas 


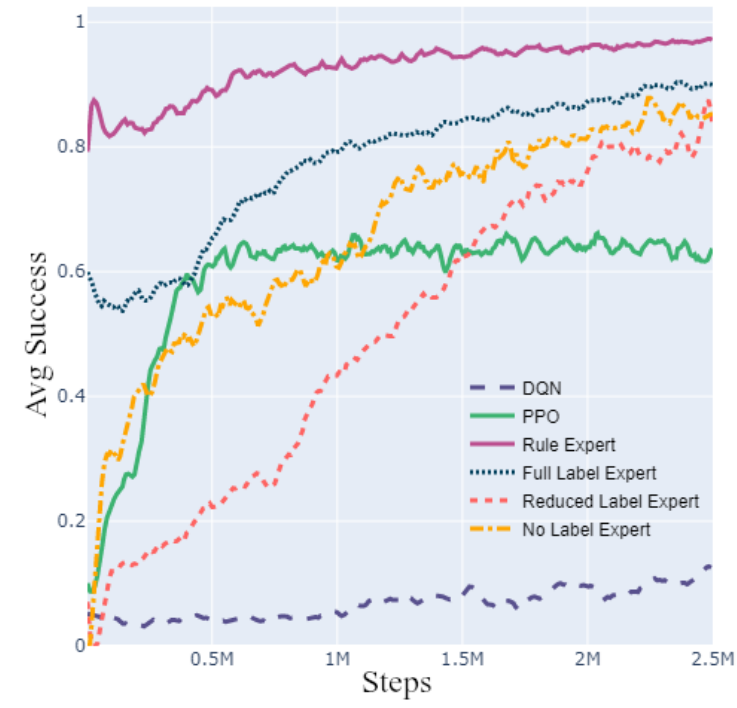

Figure 4: Average Success Rates of our methods trained on in-domain data over the course of 2.5 million training steps.

the NLE acts randomly according to a smaller, potentially higher-quality, set of actions which have high correspondence scores.

Finally, the graphs in Figure 4 indicate that none of the agents fully converge after the training step limit, although RE and FLE plateau. It is possible that after significantly more steps even DQN would converge to the ceiling performance of the Rule DM - but all our methods are considerably more sample efficient.

RoFL Training Table 1 (bottom) shows evaluation results of DQfD agents trained with RoFL fine-tuning. All weak experts improve with RoFL, especially the RLE which records an $8 \%$ jump in Success Rate. We also include the performance of the final fine-tuned FFN classifier, whose improvement over its original incarnation (15\% higher Success Rate) demonstrates that fine-tuning helps narrow the domain gap between data and the RL environment.

In addition to Table 1, Figure 5 shows DM performance over the course of training. RoFL dramatically improves both the performance and convergence rate of the RLE, indicating a domain gap between the reduced label data and the sets of environment actions. RoFL improves the FLE early in training, but this gain tails off after 1 million steps - possibly due to the relative strength of the expert. The trend for NLE-R is more ambiguous, falling behind its standard DQfD counterpart before catching up to its performance. RoFL seems

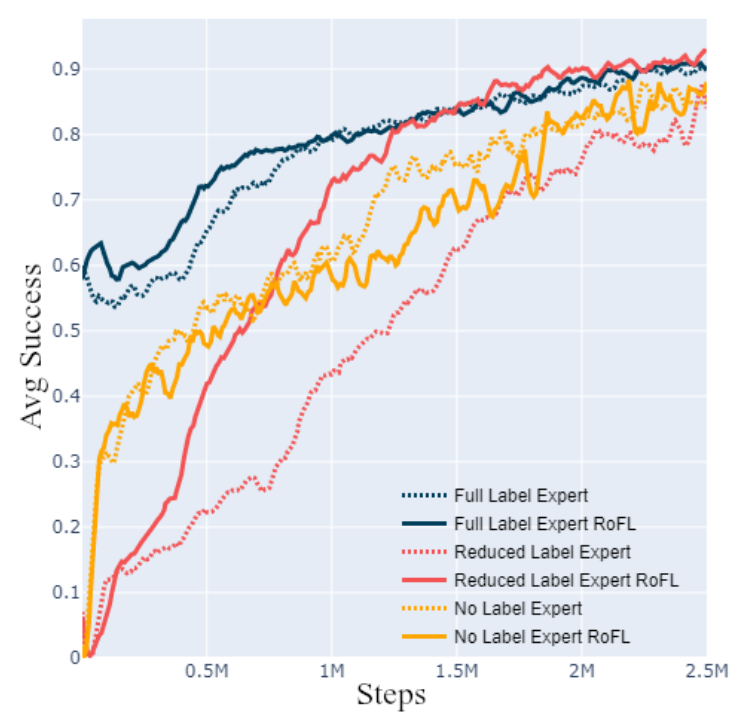

Figure 5: Average Success Rate of RL agents over the course of 2.5 million training steps, with and without RoFL fine-tuning. Agents were evaluated every 2000 steps on 100 evaluation dialogs. Experts were trained on in-domain data.

to lead to the greatest gains when the expert initially struggles.

Out-of-Domain Weak Experts The weakest experts that we evaluate were trained on out-ofdomain data. The OOD RLE, trained on Microsoft E2E and Frames, scores 53 F1 on a reduced label MultiWOZ test set, while the OOD NLE, trained on the same datasets, unannotated, only manages $41 \mathrm{~F} 1$ on the test set. Results for OOD approaches trained with and without RoFL are shown in Table 2, with training graphs in Figure 6.

Even without RoFL, the OOD RLE guides the DQfD agent to performance rates comparable to its in-domain counterpart. This indicates that even reduced labels learned on the OOD data provide the agent with enough clues to correctly satisfy some user goals. With RoFL, the OOD RLE surpasses the Success Rate of the in-domain system, and is only marginally worse than the fine-tuned in-domain expert. This shows that with RoFL we can learn a competitive DM in a challenging multi-domain environment while only using unannotated data from other dialog tasks.

RoFL leads to the greatest gain with the OOD NLE. Without fine-tuning, it scores a measly $26 \%$ Success Rate (although it should be noted that this is still higher than DQN), compared to $86 \%$ when the expert is trained on in-domain sentences. This illustrates the clear difference between the lan- 


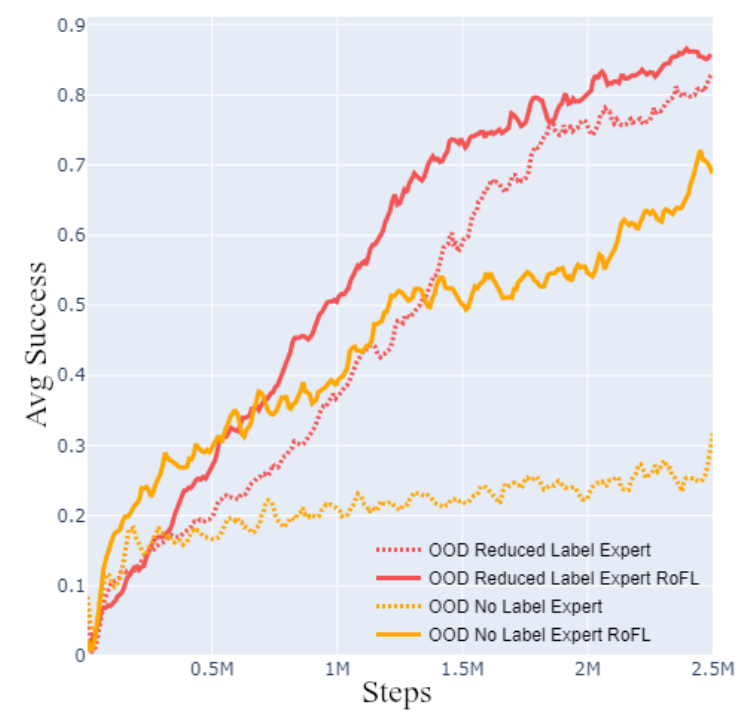

Figure 6: Average Success Rates of Reduced and No Label experts trained on out-of-domain data over the course of 2.5 million training steps, with and without RoFL fine-tuning.

\begin{tabular}{l|c|c|c|c}
\hline & Turns & Inform & Match & Success \\
\hline RLE & 7.60 & 80.33 & 87.30 & 85.00 \\
NLE & 16.27 & 40.00 & 27.15 & 26.55 \\
RLE+R & $\mathbf{6 . 6 4}$ & $\mathbf{8 5 . 0 0}$ & $\mathbf{8 9 . 0 3}$ & $\mathbf{9 1 . 0 0}$ \\
NLE+R & 9.94 & 70.00 & 62.64 & 68.90 \\
\hline
\end{tabular}

Table 2: Results of out-of-domain weak experts with and without RoFL training, using an agenda-based user-simulator for 1000 evaluation dialogs. Reported scores are average number of Turns, Inform F1, Match Rate, and Success Rate.

guage in the in- and out-of-domain data. With RoFL, OOD NLE is able to update its weights to adapt to the language of the environment, outperforming the unaltered expert's Success Rate by $35 \%$. This improvement holds true throughout training, as shown in Figure 6. The graph also shows that OOD NLE+R has not started to converge after 2.5 million training steps; it is likely that with more training it would perform similarly to the in-domain NLE DM.

\section{Conclusions and Future Work}

In this paper, we have shown that weak demonstrations can be leveraged to learn an accurate dialog manager with Deep Q-Learning from Demonstrations in a challenging multi-domain environment. We established that expert demonstrators can be trained on labeled, reduced-labeled, and unlabeled data and still guide the RL agent by means of their respective auxiliary losses. Evaluation has shown that all experts exceeded the performance of reinforcement and supervised learning baselines, and in some cases even approached the results of a hand-crafted rule-based dialog manager.

Furthermore, we introduced Reinforced Finetune Learning (RoFL) a DAgger-inspired extension to DQfD which allows a pre-trained expert to adapt to an RL environment on-the-fly, bridging the domain-gap. Our experiments show that RoFL training is beneficial across different sources of demonstration data, boosting both the rate of convergence and final system performance. It even enables an expert trained on unannotated out-ofdomain data to guide an RL dialog manager in a challenging environment.

In future, we want to continue to investigate the possibility of using even weaker demonstrations. Since our No Label Expert is trained on unannotated data, it would be interesting to leverage large and noisy conversational datasets drawn from message boards or movie subtitles, and to see how RoFL training fares with such a significant domain gap between the data and the RL environment.

\section{Acknowledgements}

We thank Ignacio Iacobacci and Gerasimos Lampouras for their valuable suggestions.

\section{References}

Yusuf Aytar, Tobias Pfaff, David Budden, Thomas Paine, Ziyu Wang, and Nando de Freitas. 2018. Playing Hard Exploration Games by Watching YouTube. In Advances in Neural Information Processing Systems, pages 2930-2941.

Johan Bos, Ewan Klein, Oliver Lemon, and Tetsushi Oka. 2003. DIPPER: Description and Formalisation of an Information-State Update Dialogue System Architecture. In Proceedings of the Fourth SIGdial Workshop of Discourse and Dialogue, pages 115-124.

Samuel R Bowman, Gabor Angeli, Christopher Potts, and Christopher D Manning. 2015. A Large Annotated Corpus for Learning Natural Language Inference. arXiv preprint arXiv:1508.05326.

Paweł Budzianowski, Tsung-Hsien Wen, Bo-Hsiang Tseng, Iñigo Casanueva, Ultes Stefan, Ramadan Osman, and Milica Gašić. 2018. MultiWOZ A Large-Scale Multi-Domain Wizard-of-Oz Dataset for Task-Oriented Dialogue Modelling. In Proc. of EMNLP 2018, pages 5016-5026. 
Kai-Wei Chang, Akshay Krishnamurthy, Alekh Agarwal, John Langford, and Hal Daumé III. 2015. Learning to Search Better Than Your Teacher. arXiv preprint arXiv:1502.02206v2.

Kyunghyun Cho, Bart Van Merriënboer, Dzmitry Bahdanau, and Yoshua Bengio. 2014. On the Properties of Neural Machine Translation: Encoder-Decoder Approaches. arXiv preprint arXiv:1409.1259.

Jacob Devlin, Ming-Wei Chang, Kenton Lee, and Kristina Toutanova. 2018. BERT: Pre-training of Deep Bidirectional Transformers for Language Understanding. arXiv preprint arXiv:1810.04805.

Layla El Asri, Hannes Schulz, Shikhar Sharma, Jeremie Zumer, Justin Harris, Emery Fine, Rahul Mehrotra, and Kaheer Suleman. 2017. Frames: A Corpus for Adding Memory to Goal-oriented Dialogue Systems. In Proceedings of the 18th Annual SIGdial Meeting on Discourse and Dialogue, pages 207-219, Saarbrücken, Germany. Association for Computational Linguistics.

Mehdi Fatemi, Layla El Asri, Hannes Schulz, Jing He, and Kaheer Suleman. 2016. Policy Networks with Two-stage Training for Dialogue Systems. arXiv preprint arXiv:1606.03152.

Jianfeng Gao, Michel Galley, Lihong Li, et al. 2019. Neural Approaches to Conversational AI. Foundations and Trends $\mathrm{R}$ in Information Retrieval, 13(23):127-298.

Gabriel Gordon-Hall, Philip John Gorinski, Gerasimos Lampouras, and Ignacion Iacobacci. 2020. Show Us the Way: Learning to Manage Dialog from Demonstrations. In The Eight Dialog System Technology Challenge (DSTC-8) at AAAI 2020.

Todd Hester, Matej Vecerík, Olivier Pietquin, Marc Lanctot, Tom Schaul, Bilal Piot, Andrew Sendonaris, Gabriel Dulac-Arnold, Ian Osband, John Agapiou, Joel Z. Leibo, and Audrunas Gruslys. 2017. Learning from Demonstrations for Real World Reinforcement Learning. CoRR, abs/1704.03732v4.

Günter Klambauer, Thomas Unterthiner, Andreas Mayr, and Sepp Hochreiter. 2017. Self-Normalizing Neural Networks. In Advances in neural information processing systems, pages 971-980.

Sungjin Lee, Qi Zhu, Ryuichi Takanobu, Xiang Li, Yaoqin Zhang, Zheng Zhang, Jinchao Li, Baolin Peng, Xiujun Li, Minlie Huang, and Jianfeng Gao. 2019. ConvLab: Multi-Domain End-to-End Dialog System Platform. In Proc. of ACL 2019.

Xiujun Li, Yun-Nung Chen, Lihong Li, and Jianfeng Gao. 2017. End-to-End Task-Completion Neural Dialogue Systems. CoRR, abs/1703.01008.

Xiujun Li, Sarah Panda, Jingjing Liu, and Jianfeng Gao. 2018. Microsoft Dialogue Challenge: Building End-to-End Task-Completion Dialogue Systems. arXiv preprint arXiv:1807.11125.
Zachary Lipton, Xiujun Li, Jianfeng Gao, Lihong Li, Faisal Ahmed, and Li Deng. 2018. BBQ-Networks: Efficient Exploration in Deep Reinforcement Learning for Task-oriented Dialogue Systems. In Proc. of AAAI 2018.

Diane J. Litman and James F. Allen. 1987. A Plan Recognition Model for Subdialogues in Conversations. Cognitive Science, 11(2):163-200.

Bing Liu and Ian Lane. 2017. Iterative Policy Learning in End-to-End Trainable Task-Oriented Neural Dialog Models. In 2017 IEEE Automatic Speech Recognition and Understanding Workshop (ASRU), pages 482-489. IEEE.

Bing Liu, Gokhan Tur, Dilek Hakkani-Tur, Pararth Shah, and Larry Heck. 2018. Dialogue Learning with Human Teaching and Feedback in End-to-End Trainable Task-Oriented Dialogue Systems. arXiv preprint arXiv:1804.06512.

Liyuan Liu, Haoming Jiang, Pengcheng He, Weizhu Chen, Xiaodong Liu, Jianfeng Gao, and Jiawei Han. 2019. On the Variance of the Adaptive Learning Rate and Beyond. arXiv preprint arXiv:1908.03265.

Keting Lu, Shiqi Zhang, and Xiaoping Chen. 2019. Goal-Oriented Dialogue Policy Learning from Failures. arXiv preprint arXiv:1808.06497v2.

Volodymyr Mnih, Koray Kavukcuoglu, David Silver, Andrei A. Rusu, Joel Veness, Marc G. Bellemare, Alex Graves, Martin Riedmiller, Andreas K. Fidjeland, Georg Ostrovski, Stig Petersen, Charles Beattie, Amir Sadik, Ioannis Antonoglou, Helen King, Dharshan Kumaran, Daan Wierstra, Shane Legg, and Demis Hassabis. 2015. Human-level Control Through Deep Reinforcement Learning. Nature, 518(7540):529-533.

Jeffrey Pennington, Richard Socher, and Christopher D. Manning. 2014. GloVe: Global Vectors for Word Representation. In Proc. of EMNLP 2014, pages 1532-1543.

Abhinav Rastogi, Xiaoxue Zang, Srinivas Sunkara, Raghav Gupta, and Pranav Khaitan. 2019. Towards Scalable Multi-domain Conversational Agents: The Schema-guided Dialogue Dataset. arXiv preprint arXiv:1909.05855.

Stéphane Ross, Geoffrey Gordon, and Drew Bagnell. 2011. A Reduction of Imitation Learning and Structured Prediction to No-regret Online Learning. In Proceedings of the fourteenth international conference on artificial intelligence and statistics, pages 627-635.

Tim Salimans and Richard Chen. 2018. Learning Montezuma's Revenge from a Single Demonstration. arXiv preprint arXiv:1812.03381.

Jost Schatzmann, Blaise Thomson, Karl Weilhammer, Hui Ye, and Steve Young. 2007. Agenda-Based 
User Simulation for Bootstrapping a POMDP Dialogue System. In Proc. NAACL (Short Papers), pages 149-152.

Tom Schaul, John Quan, Ioannis Antonoglou, and David Silver. 2015. Prioritized Experience Replay. arXiv preprint arXiv:1511.05952.

John Schulman, Filip Wolski, Prafulla Dhariwal, Alec Radford, and Oleg Klimov. 2017. Proximal policy optimization algorithms. arXiv preprint arXiv:1707.06347.

Pararth Shah, Dilek Hakkani-Tür, Gokhan Tür, Abhinav Rastogi, Ankur Bapna, Neha Nayak, and Larry Heck. 2018. Building a Conversational Agent Overnight with Dialogue Self-Play. arXiv preprint arXiv:1801.04871.

Pei-Hao Su, Milica Gasic, Nikola Mrksic, Lina RojasBarahona, Stefan Ultes, David Vandyke, TsungHsien Wen, and Steve Young. 2016. Continuously Learning Neural Dialogue Management. arXiv preprint arXiv:1606.02689.

Richard S Sutton and Andrew G Barto. 2018. Reinforcement Learning: An Introduction. MIT press.

Ryuichi Takanobu, Hanlin Zhu, and Minlie Huang. 2019. Guided Dialog Policy Learning: Reward Estimation for Multi-Domain Task-Oriented Dialog. arXiv preprint arXiv:1908.10719.

Hado Van Hasselt, Arthur Guez, and David Silver. 2016. Deep Reinforcement Learning with Double Q-Learning. In Proc. of AAAI 2016.

Marilyn A Walker. 2000. An Application of Reinforcement Learning to Dialogue Strategy Selection in a Spoken Dialogue System for Email. JAIR, 12:387416.

Ziyu Wang, Tom Schaul, Matteo Hessel, Hado Van Hasselt, Marc Lanctot, and Nando De Freitas. 2015. Dueling Network Architectures for Deep Reinforcement Learning. CoRR, abs/1511.06581.

Jason D Williams, Kavosh Asadi, and Geoffrey Zweig. 2017. Hybrid Code Networks: Practical and Efficient End-to-End Dialog Control with Supervised and Reinforcement Learning. arXiv preprint arXiv:1702.03274.

Steve J. Young, Milica Gasic, Blaise Thomson, and Jason D. Williams. 2013. POMDP-Based Statistical Spoken Dialog Systems: A Review. Proc. of IEEE, 101(5):1160-1179.

\section{A Model Hyperperameters}

Below we list the hyperparameters used for our reinforcement learning agents and the expert models used to generate demonstrations.
For No Label Expert RoFL we treat dialogs with a final reward $r>=t h$ as positive, and those with reward $r<t h$ as negative examples, and treat the individual user-agent utterance pairs accordingly.

General Hyperparameters Unless otherwise stated in specific expert sections, all of our agents use below hyperparameters, where applicable:

\begin{tabular}{l|l}
\hline Steps & $2,500,000$ \\
Pre-training steps $d$ & 2,000 dialogs \\
$\epsilon$ start value & 0.1 \\
$\epsilon$ end value & 0.01 \\
$\epsilon$ decay rate & $\begin{array}{l}\text { Linear over 500,000 } \\
\text { steps }\end{array}$ \\
Discount factor $\gamma$ & 0.9 \\
Policy net $\pi$ & $1 \times 100$ d hidden layer, \\
& ReLU activation \\
Learning rate & 0.01 \\
Target network update & 10,000 steps \\
period $\tau$ & \\
L2 reg. weight & $10^{-5}$ \\
Max replay size & 100,000 \\
Prioritized replay $\alpha$ & 0.6 \\
Prioritized replay $\epsilon_{p}$ & 0.001 \\
Prioritized replay $\epsilon_{d}$ & 1.0 \\
Prioritized replay $\beta_{0}$ & 0.4 \\
\hline
\end{tabular}

\section{Full Label Expert}

\begin{tabular}{l|l}
\hline Input & $\begin{array}{l}\text { 392d binary state } \\
\text { Expert network }\end{array}$ \\
Output & $\begin{array}{l}\text { ReLU, dropout }=0.1 \\
\text { Single-label softmax } \\
\text { over 300 actions }\end{array}$ \\
\hline \hline
\end{tabular}

\begin{tabular}{l|l}
\hline \hline Penalty $c$ & 0.8 \\
Reward threshold $t h$ & 70 \\
Finetune interval $k$ & 2,000 steps \\
\hline
\end{tabular}

Reduced Label Expert

Input

300d pre-trained GloVe embeddings

Expert network 1x128d GRU layer, input dropout $=0.1$

Output Multi-label sigmoid of 3 reduced actions, with threshold $=0.5$

\begin{tabular}{l|l}
\hline \hline Pre-training steps $d$ & 3,000 dialogs \\
$\epsilon$ start value & 0.2 \\
Penalty $c$ & 1.0 \\
Reward threshold $t h$ & 70 \\
Finetune interval $k$ & 15,000 steps \\
\hline
\end{tabular}


No Label Expert

\begin{tabular}{l|l}
\hline Input & $\begin{array}{l}\text { BERT } \\
\text { beddings for used em- }\end{array}$ \\
& $\begin{array}{l}\text { agent utterances, con- } \\
\text { catenated 1536d }\end{array}$ \\
6 Expert network & $\begin{array}{l}256, \text { linear layers, 512, 64, 32, 16 } \\
\text { dims, SELU activa- }\end{array}$ \\
& $\begin{array}{l}\text { tions } \\
\text { Binary output of re- } \\
\text { sponse appropriateness } \\
\text { with threshold } \rho=0.9\end{array}$ \\
Output & $\begin{array}{l}0.8 \\
70\end{array}$ \\
\hline \hline Penalty $c$ & 15,000 steps \\
Reward threshold $t h$ & \\
Finetune interval $k$ & \\
\hline
\end{tabular}

